\title{
Corrector commands
}

The various error correctors were run using a Makefile. In the following code listing, the variable ' $\$<$ ' represents the input file, ' $\$ @$ ' represents the output file, ' $\$(\mathrm{NTH})$ ' is the number of CPU cores to use, ' $\$\left(J F \_S I Z E\right)$ ' is an estimate of the number of $k$-mers in the reads, and '\$(GENOME_SIZE)' is the size of the genome. Some of the software below use multiple commands (e.g. Quake and QuorUM). Both of them have easier to use wrapper scripts.

Echo

python ErrorCorrection · py - ncpu $\$(\mathrm{NCPU})-\mathrm{o} \$ \$ \$<$

HiTec

hitec $\$<\$ @ \$$ (GENOME_SIZE) 1

\section{QuorUM}

quorum_create_database - $\mathrm{t} \$(\mathrm{NTH})$ - $\mathrm{s}$ \$(JF_SIZE) -b $8-\mathrm{m} 24-\mathrm{q} 37$-o mer_db.jf $\$<$ quorum_error_correct_reads --contaminant share/adapter.jf $-\mathrm{m} 1$-s 1 -g 1 -a 3 ।

$-\mathrm{t} \$(\mathrm{NTH})-\mathrm{w} 10-\mathrm{e} 3-\mathrm{o}$ quorum/corrected_tmp mer_db.jf $\$<$

\section{Musket}

musket $-\mathrm{k} 28$ \$(JF_SIZE) -o \$@.tmp - $\$$ \$(NTH) - maxtrim $50 \$<$

\section{SGA}

sga index $-\mathrm{a}$ ropebwt $-\mathrm{t} \$(\mathrm{NTH})-\mathrm{p}$ prefix $\$<$

sga correct - p prefix -t $\$(N T H)$ - discard - metrics metrics -o $\$ @ \$<$

\section{Racer}

RACER \$< \$@ \$(GENOME_SIZE)

Quake For Quake, the file 'fastq_list ' contains the names of the fastq files with paired reads.

count-qmers -k 15 -f $\$<>$ qmer_counts

cov_model.py qmer_counts $>$ cutoff.log

correct $-\mathrm{f}$ fastq_list $-\mathrm{k} 15-\mathrm{c}$ 'cat cutoff' $-\mathrm{m}$ qmer_counts $-\mathrm{p} \$(\mathrm{NTH})$

\section{Assembler commands}

The SOAPdenovo assembler was run as follows:

SOAPdenovo-63mer all $-\mathrm{s}$ soap. conf $-\mathrm{d} \quad 0-\mathrm{K} 31-\mathrm{R}-\mathrm{o}$ output $-\mathrm{N}$ \$(GENOME_SIZE) $-\mathrm{p} \$(\mathrm{NTH})$ with the following configuration file "soap.conf":

\#maximal read length

max_rd_len $=101$

[LIB ]

\#average insert size

avg_ins $=180$

\#if sequence needs to be reversed

reverse_seq $=0$

\#in which part(s) the reads are used

as $\mathrm{m}_{\text {_flags }}=3$ 


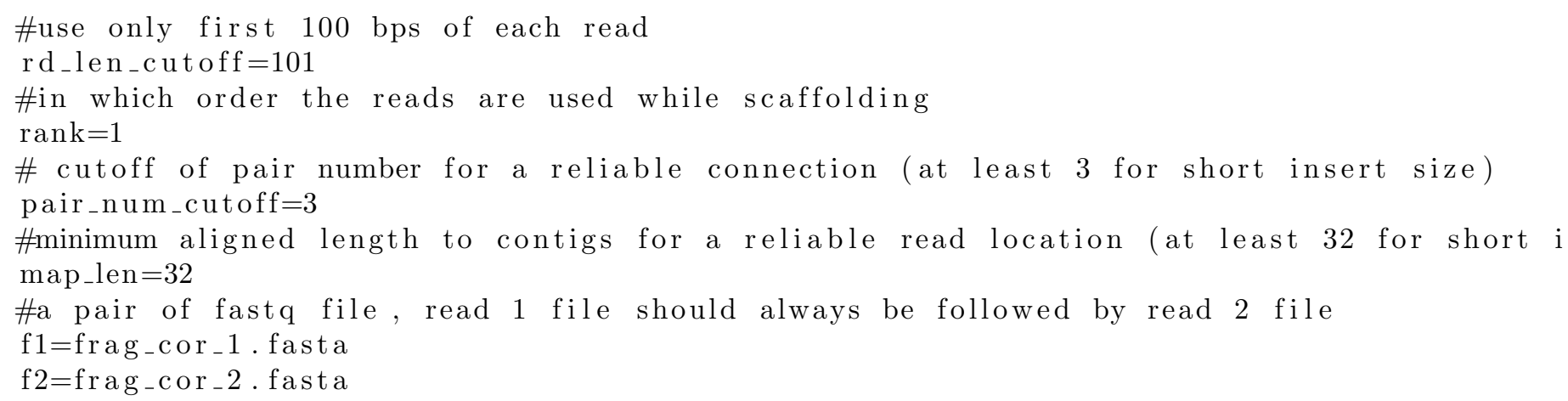

To run MaSuRCA, the following configuration file was used:

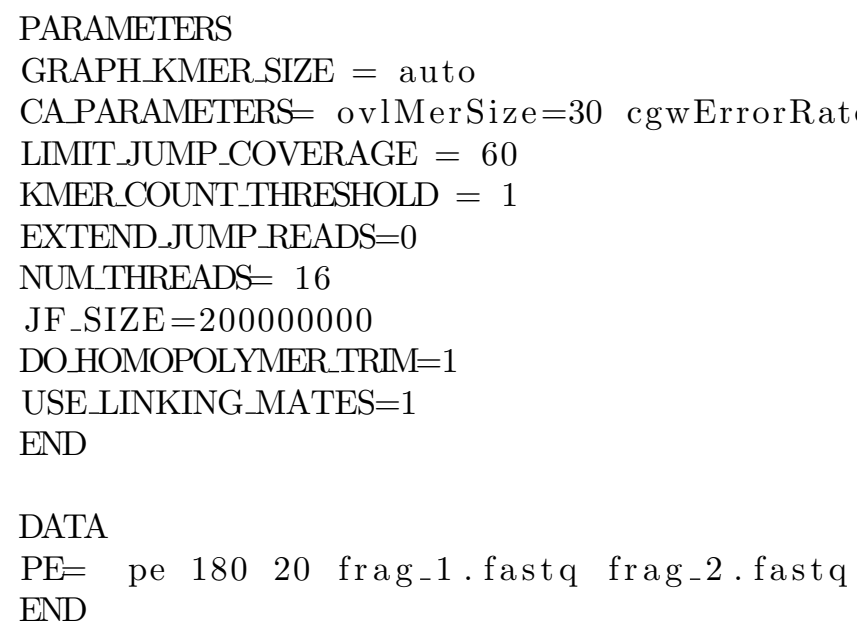

Then the resulting "assemble.sh" script was modified to not run QuorUM and instead take already error-corrected reads as input. 